\title{
Microscopic theory of the rhombohedral phase and transition to the monoclinic phase of solid $\mathbf{C}_{70}$
}

\author{
A. V. Nikolaev* and K. H. Michel \\ Departement Natuurkunde, Universitaire Instelling Antwerpen, 2610 Antwerpen, Belgium
}

(Received 24 June 1996)

\begin{abstract}
Starting from a model of microscopic interactions between $\mathrm{C}_{70}$ molecules, we have developed a theory which describes the orientational dynamics and its coupling to lattice displacements in the rhombohedral phase of $\mathrm{C}_{70}$ fullerite. The Landau free energy is calculated. We obtain a first-order phase transition to a monoclinic structure with the space group $P 2_{1} / \mathrm{m}$. The transition is driven by the condensation of orientational quadrupoles at the $F$ point of the Brillouin zone of the rhombohedral lattice. We find no evidence that the monoclinic structure is connected with the freezing in of orientations around the fivefold molecular axis. We calculate the lattice strains that are associated with the transition to the monoclinic structure. The theory is compared with a range of experimental data on the phase transition. [S0163-1829(96)07742-9]
\end{abstract}

\section{INTRODUCTION}

Solid $\mathrm{C}_{70}$ (Ref. 1) undergoes a sequence of structural phase transitions. $^{2-4}$ At high temperature $\gtrsim 350 \mathrm{~K}$, solid $\mathrm{C}_{70}$ forms an orientationally disordered phase ${ }^{5}$ with facecentered-cubic (fcc) lattice (space group $F m \overline{3} m$ ). Depending on the treatment of the sample, also a hexagonal closepacked structure exists. ${ }^{1,2,6,7}$ Upon cooling from the fcc phase, a transition to a rhombohedral phase (space group $R \overline{3} m$ ) occurs, followed by a transition to a monoclinic phase around $295 \mathrm{~K}^{2-4}$ Unlike in solid $\mathrm{C}_{60}$ where the transition from the $F m \overline{3} m$ to the $P a \overline{3} m$ phase is essentially a phenomenon of orientational ordering, ${ }^{8-10}$ while the centers of mass of the molecules still occupy an fcc lattice, the transition from the cubic to the rhombohedral phase in solid $\mathrm{C}_{70}$ is due to a coupling of orientational quadrupoles of $T_{2 g}$ symmetry to trigonal shear strains. ${ }^{11,12}$ Such a bilinear coupling which is characteristic for ferroelastic transitions, ${ }^{13}$ is favored by the elongated shape of the $\mathrm{C}_{70}$ molecule. Important insight can be gained by molecular-dynamics simulations in solid $\mathrm{C}_{70} \cdot{ }^{14}$ Molecular dynamics shows that in the rhombohedral phase the molecules are on the average oriented along a [111] direction of the former cubic phase and that the unit cell is stretched along this direction. It is also found that the rhombohedral phase is followed by a monoclinic phase ${ }^{14}$ at lower temperature.

The $\mathrm{C}_{70}$ molecule has symmetry $D_{5 h}$ with a long fivefold axis. ${ }^{15}$ As was noticed before, in the rhombohedral phase the $\mathrm{C}_{70}$ molecules effectively behave as solid objects of $D_{\infty h}$ symmetry performing fast rotations about long axes. ${ }^{11,12,16}$ The phase transition to the monoclinic phase was initially associated with orientational ordering of $\mathrm{C}_{70}$ molecules where the rotations around the fivefold axis are frozen-in. ${ }^{2}$ This conclusion prompted several investigations where rotator functions belonging to the manifold $l=5$ were chosen as order parameters for the monoclinic phase. ${ }^{17,18}$ However, this approach predicts a space group $\mathrm{Pbnm},{ }^{19}$ while the experimentally identified symmetry group is $P 2_{1} / m .^{3,20,21}$

In this paper we propose a theory of the transition based on a microscopic model which indicates that quadrupolar interactions $(l=2)$ remain the strongest ones driving the transition, while the interactions connected with $l=5$ are negligible and unimportant. Our results are consistent with all experimental data on the phase transition from the rhombohedral to the monoclinic phase.

\section{ORIENTATIONAL COORDINATES AND INTERACTION POTENTIAL}

In the present section we will derive the interaction potential for a system of $N \mathrm{C}_{70}$ molecules in the rhombohedral phase. A convenient way to treat the orientation-dependent properties of nonlinear molecules in crystals is to use rotator functions. $^{22-24}$ The rotator functions take into account the symmetry of the molecule and of the site. Rotator functions for the cubic phase of solid $\mathrm{C}_{70}$ were given in Ref. 12. Here we first extend this concept to the rhombohedral phase.

We start with a molecule in its standard orientation ${ }^{15}$ with respect to a rectangular coordinate system. The molecule is centered at the origin, its fivefold axis is taken as $z$ axis, the $(x, y)$ plane is a symmetry plane. The Cartesian coordinates of the molecule, taken as a rigid body, are specified in Ref.15. We classify the atoms (also called $C$ centers) of the molecule in (001) planes. We consider five groups of atoms labeled by an index $\lambda(\lambda=1-5$, as shown in Fig. 1$)$. There are ten atoms in the equatorial plane $z(\lambda=1)=0$, ten atoms in each of the planes $\pm z(\lambda), \lambda=2,3$ and five atoms in each of the planes $\pm z(\lambda), \lambda=4,5$. To describe the intermolecular interaction due to charge-density variations on $C-C$, bonds ${ }^{25}$ we introduce "double bond" centers of interaction (called $D$ centers) and "intermediate bond" centers (called $I$ centers). There are five $D$ centers in each of the planes $\pm z(6)$ and in planes $\pm z(7)$. Finally there are five $I$ centers in each of the planes $\pm z(8)$ and ten in each plane $\pm z(9)$ (see also Table I of Ref.12). The centers in the layers are labeled by indices $\nu(\lambda)$, the correspondent polar coordinates of the centers are $\left[d(\lambda), \Omega^{\prime}(\nu(\lambda)]\right.$, where $d$ is distance from the origin to the center and where $\Omega^{\prime} \equiv\left(\theta^{\prime}, \phi^{\prime}\right)$ are the polar angles. We then calculate the molecular structure coefficients $^{10}$ 


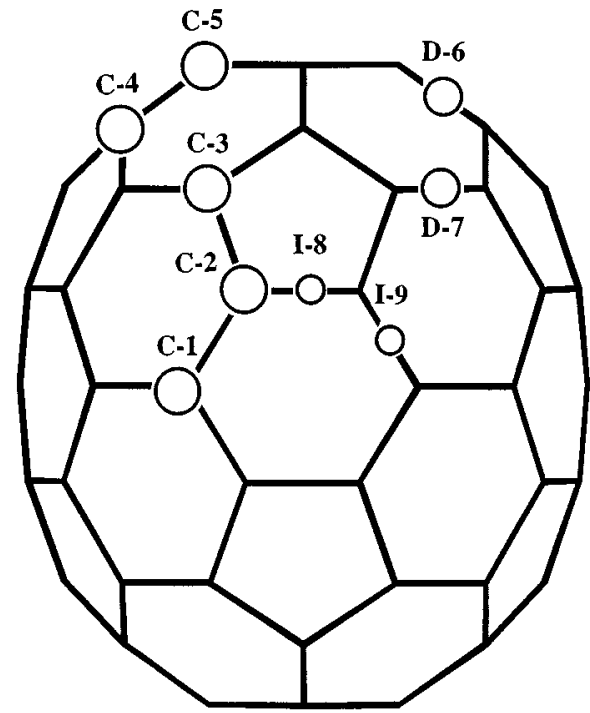

FIG. 1. The $\mathrm{C}_{70}$ molecules with $C$ (atomic $\lambda=1-5$ ), $D$ (double bond $\lambda=6,7$ ), and $I$ (intermediate, $\lambda=8,9)$ interaction centers.

$$
c_{l}^{m}(\lambda)=\sum_{\nu=1}^{n(\lambda)} Y_{l}^{m}\left(\Omega^{\prime}(\nu(\lambda)) .\right.
$$

Here $n(\lambda)$ is the number of centers in group $\lambda$. Symmetry of the molecule implies that the coefficients $c_{l}^{m}(\lambda)$ are different from zero for $m=0, \pm 5, \pm 10, \ldots$ and $(l-m)$ even. ${ }^{11,5,12}$ For $l=0,2,4,6$, and 8 , only the coefficients $c_{l}^{0}(\lambda)$ are different from zero. They are quoted in Table I of Ref.12. Since $m=0$, the azimuthal angle $\phi$ is irrelevant, the molecule is spinning around its fivefold axis.

In the rhombohedral phase, the site symmetry is described by the point group $D_{3 d}$. The corresponding symmetry adapted functions (SAF's) are tabulated in Ref. 26:

$$
S_{l}^{\tau}(\Omega)=\sum_{m=-l}^{l} Y_{l}^{m}(\Omega) \alpha_{l}^{m \tau}
$$

Here $\tau \equiv(\Gamma, \rho, \delta)$, where $\Gamma$ refers to the irreducible representations of the group $D_{3 d}, \rho$ labels the subspaces of $\Gamma$ if $\Gamma$ occurs more than once for a given $l, \delta$ labels the components of $\Gamma$.

The site symmetry adapted functions are defined with respect to the crystal fixed system of axes shown in Fig. 2. In particular the $z$ axis coincides with the threefold axis of $D_{3 d}$. In order to relate the site symmetry and the molecular symmetry, we construct the rotator functions by following the procedure of Ref. 12. We start from a situation where the molecular system of axes coincides with the crystal fixed

TABLE I. Interaction potential parameters between $C, D, I$ centers of interaction.

\begin{tabular}{lccccccc}
\hline \hline & units & $C, C$ & $C, D$ & $C, I$ & $D, D$ & $D, I$ & $I, I$ \\
\hline$C_{\lambda \lambda^{\prime}}^{(1)}$ & $10^{6} \mathrm{~K}$ & 6.24 & 6.24 & 0.84 & 6.24 & 0.84 & 0.114 \\
$C_{\lambda \lambda^{\prime}}^{(2)}$ & $\AA^{-1}$ & 3.6 & 3.4 & 3.5 & 3.2 & 3.3 & 3.4 \\
$B_{\lambda \lambda^{\prime}}$ & $10^{4} \mathrm{~K} \times \AA^{6}$ & 4.24 & 0 & 0 & 0 & 0 & 0 \\
\hline \hline
\end{tabular}

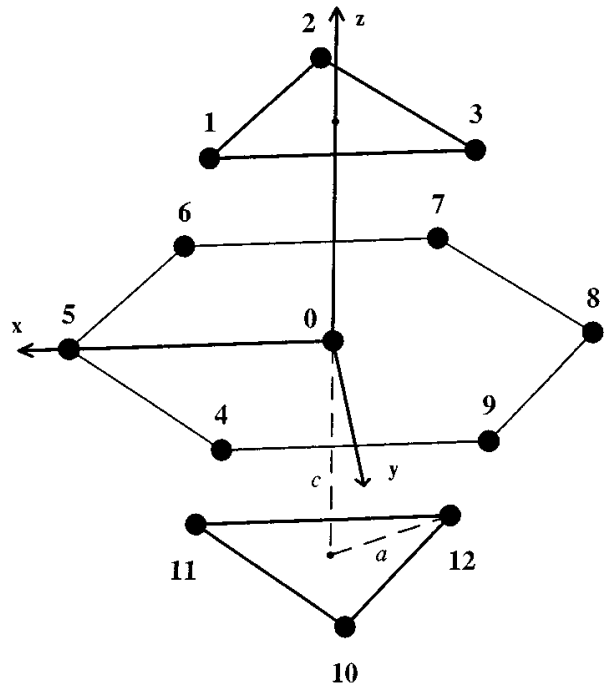

FIG. 2. The nearest neighbors of a $\mathrm{C}_{70}$ molecule (0) in rhombohedral phase. Indices 4-9 refer to sites laying on the base plane, $1-3$ sites lay on the plane situated above the base plane, and 10-12, below the base plane. Lattice constants $a$ and $c$ are indicated.

system of axes. Then the molecule is tilted away from the standard position by a rotation $R(\omega), \omega$ stands for the Euler angles which specify the orientation of the molecule with respect to crystal fixed system of axes. The corresponding rotator functions are then given by

$$
U_{l}^{\tau}(\omega, \lambda)=c_{l}^{0}(\lambda) \sum_{m} D_{l}^{0 m}(\omega) \alpha_{l}^{m \tau}
$$

Here the coefficients $\alpha_{l}^{m \tau}$ refer to the site point group $D_{3 d}$. The Wigner matrix $D_{l}^{0 m}$ is given by

$$
D_{l}^{0 m}(\omega)=(-1)^{m} \sqrt{\frac{4 \pi}{2 l+1}} Y_{l}^{m}(\beta, \alpha)^{*} .
$$

Here $\beta$ and $\alpha$ are polar angles of the fivefold axis of the molecule. As we have seen the third Euler angle $\gamma=\phi$, which specifies the rotation of the molecule about the fivefold axis, is irrelevant. It should be noted that rotator functions with $l=5$ would restore the azimuthal $\phi$ dependence. As we will see later, the corresponding contributions to the intermolecular potential are negligibly small and can be omitted. In the following it will be convenient to use rotator functions which are independent on the group index $\lambda$ and hence we define

$$
U_{l}^{\tau}(\omega)=\sum_{m} D_{l}^{0 m}(\omega) \alpha_{l}^{m \tau}
$$

The coefficients $c_{l}^{0}(\lambda)$ which occur in $U_{l}^{\tau}(\omega, \lambda)$ will then be counted by the expansion coefficients of the intermolecular potential (see below). In Appendix A we quote relevant rotator functions of the group $D_{3 d}$.

The total intermolecular potential is given by a sum of potentials between interaction centers belonging to different molecules 


$$
V=\frac{1}{2} \sum_{\vec{n} n^{\prime}} \sum_{\lambda \lambda^{\prime}} \sum_{\nu \nu^{\prime}} V\left(\vec{n}, \lambda, \nu ; \vec{n}^{\prime}, \lambda^{\prime}, \nu^{\prime}\right)
$$

The potential $V\left(\vec{n}, \lambda, \nu ; \vec{n}^{\prime}, \lambda^{\prime}, \nu^{\prime}\right)$ depends on the distance $r$ between the center $\nu(\lambda)$ on molecule $\vec{n}$ and the center $\nu^{\prime}\left(\lambda^{\prime}\right)$ on molecule $\vec{n}^{\prime}$. One has

$$
r=\left|\vec{R}(\vec{n}, \lambda, \nu)-\vec{R}\left(\vec{n}^{\prime}, \lambda^{\prime}, \nu^{\prime}\right)\right|,
$$

where the position of the interaction center $\nu(\lambda)$ on the $\vec{n}$ th molecule is given by

$$
\vec{R}(\vec{n}, \lambda, \nu)=\vec{X}(\vec{n})+\vec{d}(\nu(\vec{n}, \lambda)) .
$$

Here $\vec{X}(\vec{n})$ is the center-of-mass position of the molecule and $\vec{d}$ specifies the position of the center $\nu(\lambda)$ with respect to the molecular center of mass. Here $\vec{d}(\nu(\vec{n}, \lambda))$ has polar coordinates $d(\lambda), \Omega(\nu(\vec{n}, \lambda))$ and depends on the orientation of the molecule. The positions $\{\vec{X}(\vec{n})\}$ form a rigid rhombohedral lattice. Expanding the potential $V$ in terms of rotator functions, we obtain

$$
V=V^{\mathrm{RR}}+V^{R}
$$

Here $V^{\mathrm{RR}}$ refers to the rotation-rotation $(\mathrm{RR})$ dependent part of the intermolecular potential and $V^{R}$ is the crystal-field potential. Explicitly one has

$$
V^{\mathrm{RR}}=\frac{1}{2} \sum_{\vec{n} n^{\prime}} \sum_{l l^{\prime}} \sum_{\tau \tau^{\prime}} J_{l l^{\prime}}^{\tau \tau^{\prime}}\left(\vec{n}-\vec{n}^{\prime}\right) U_{l}^{\tau}(\Omega(\vec{n})) U_{l^{\prime}}^{\tau^{\prime}}\left(\Omega\left(\vec{n}^{\prime}\right)\right) .
$$

Here $\vec{n}$ stands for $\omega(\vec{n})$. The index $\tau\left(\tau^{\prime}\right)$ refers to irreducible representations of $D_{3 d}$ which are different from the unit representation. The coefficients are given by

$$
J_{l l^{\prime}}^{\tau \tau^{\prime}}\left(\vec{n}-\vec{n}^{\prime}\right)=\sum_{\lambda \lambda^{\prime}} v_{l l^{\prime}}^{\tau \tau^{\prime}}\left(\vec{n}, \lambda ; \vec{n}^{\prime}, \lambda^{\prime}\right) c_{l}^{0}(\lambda) c_{l^{\prime}}^{0}\left(\lambda^{\prime}\right)
$$

where

$$
\begin{aligned}
v_{l l^{\prime}}^{\tau \tau^{\prime}}\left(\vec{n}, \lambda ; \vec{n}^{\prime}, \lambda^{\prime}\right)= & \int d \Omega_{\nu} \int d \Omega_{\nu^{\prime}} V\left(\vec{n}, \lambda, \nu ; \vec{n}^{\prime}, \lambda^{\prime}, \nu^{\prime}\right) \\
& \times S_{l}^{\tau}\left(\Omega_{\nu}\right) S_{l^{\prime}}^{\tau^{\prime}}\left(\Omega_{\nu^{\prime}}\right)
\end{aligned}
$$

The crystal field is given by a sum of single-particle terms:

$$
V^{R}=\sum_{\vec{n}} \sum_{l} \sum_{\rho} w_{l}^{A_{1 g}, \rho} U_{l}^{A_{1 g}, \rho}(\vec{n}),
$$

where

$$
w_{l}^{A_{1 g}, \rho}=\sum_{n^{\prime}=1}^{12} \sum_{\lambda \lambda^{\prime}} c_{0}^{0}(\lambda) c_{l}^{0}\left(\lambda^{\prime}\right) w_{l}^{A_{1 g}, \rho}\left(\vec{n}, \lambda ; \vec{n}^{\prime}, \lambda^{\prime}\right)
$$

with

$$
\begin{aligned}
w_{l}^{A_{1 g}, \rho}\left(\vec{n}, \lambda ; \vec{n}^{\prime}, \lambda^{\prime}\right)= & \frac{1}{\sqrt{4 \pi}} \int d \Omega_{\nu} \int d \Omega_{\nu^{\prime}} V \\
& \times\left(\vec{n}, \lambda, \nu ; \vec{n}^{\prime}, \lambda^{\prime}, \nu^{\prime}\right) S_{l}^{A_{1 g}, \rho}\left(\Omega_{\nu}\right)
\end{aligned}
$$

The functions $S_{l}^{A_{1 g}, \rho}$ and $U_{l}^{A_{1 g}, \rho}$ are quoted in Appendix A. The crystal field is the potential experienced by a molecule at site $\vec{n}$, while its neighbors at sites $\vec{n}^{\prime}$ are taken in spherical approximation. Here we restrict ourselves to the 12 nearest neighbors in the rhombohedral lattice (see Fig. 2).

In the next section we will study collective phenomena. It is useful to rewrite the orientation-orientation dependent interaction in Fourier space. Defining

$$
\begin{gathered}
\mathcal{U}_{l}^{\tau}(\vec{q})=\frac{1}{\sqrt{N}} \sum_{\vec{n}} e^{-i \vec{q} \cdot \vec{X}(\vec{n})} U_{l}^{\tau}(\vec{n}), \\
\mathcal{J}_{l l^{\prime}}^{\tau \tau^{\prime}}(\vec{q})=\sum_{\vec{n}^{\prime}} J_{l l^{\prime}}^{\tau \tau^{\prime}}\left(\vec{n}-\vec{n}^{\prime}\right) e^{i \vec{q} \cdot\left[\vec{X}(\vec{n})-\vec{X}\left(\vec{n}^{\prime}\right)\right]},
\end{gathered}
$$

where $\vec{q}$ is the wave vector, we get for the rotation-rotation interaction

$$
V^{\mathrm{RR}}=\frac{1}{2} \sum_{\vec{q}} \sum_{l l^{\prime}} \sum_{\tau \tau^{\prime}} \mathcal{J}_{l l^{\prime}}^{\tau \tau^{\prime}}(\vec{q}) \mathcal{U}_{l}^{\tau}(\vec{q}) \mathcal{U}_{l^{\prime}}^{\tau^{\prime}}(-\vec{q})
$$

\section{ORIENTATIONAL PHASE TRANSITION}

In the following we will investigate whether the orientation-dependent interactions $V=V^{\mathrm{RR}}+V$ in the rhombohedral phase lead to an orientational phase transition. Our aim is to predict the structure of the low-symmetry phase.

We start from a phenomenological model of the intermolecular potential which is based on the interactions between the centers of type $C, D$, and $I$ on neighboring molecules. The interaction is given by a sum of repulsive Born-Mayer and attractive van der Waals potential:

$$
V\left(\vec{n}, \lambda, \nu ; \vec{n}^{\prime}, \lambda^{\prime}, \nu^{\prime}\right)=C_{\lambda \lambda^{\prime}}^{(1)} \exp \left(-C_{\lambda \lambda^{\prime}}^{(2)} r\right)-B_{\lambda \lambda^{\prime}} r^{-6},
$$

where the distance $r$ is given by expression (2.7). The potential parameters $C_{\lambda \lambda^{\prime}}^{(1)}, C_{\lambda \lambda^{\prime}}^{(2)}$, and $B_{\lambda \lambda^{\prime}}$ are specified in Table I. The potential used here is somewhat different from the one used in Ref. 12. In particular we have reduced the coefficients $C^{(1)}$ for the repulsive interactions involving $I$ centers and we have, on the other hand, retained an attractive van der Waals part only for $C$ centers. For a comparison with the results of Ref. 12, we refer the reader to the end of Sec. IV.

\section{A. Rotation-rotation interaction $V^{R R}$}

We have calculated the interaction coefficients $J_{l l^{\prime}}^{\tau \tau^{\prime}}\left(\vec{n}-\vec{n}^{\prime}\right)$ of the intermolecular potential $V^{\mathrm{RR}}$ by means of expressions (2.11) and (2.12). Here again we restrict ourselves to the 12 nearest neighbors $\vec{n}^{\prime}$ of a molecule centered at $\vec{n}$ in the rhombohedral lattice. The relevant SAF's of $E_{g}$ and $E_{u}$ symmetry are given in Appendix A. The angular 
TABLE II. Partial contribution of $C, D, I$ centers to the constant $J_{F}$, in $\mathrm{K}$

\begin{tabular}{cccccccc}
\hline \hline & $C, C$ & $C, D$ & $D, D$ & $I, C$ & $I, D$ & $I, I$ & total \\
\hline$J_{F}$ & -10017 & -19595 & -8581 & 847 & 782 & -16 & -36580 \\
\hline \hline
\end{tabular}

integrations are done numerically. We find that by far the largest matrix elements are obtained for the quadrupolar SAF's $S_{2}^{E_{g}, 3}=Y_{2}^{2, c}$ and $S_{2}^{E_{g}, 4}=-Y_{2}^{2, s}$, which form a twodimensional representation $E_{g}$ of the group $D_{3 d}$. We have estimated that the interaction coefficients $J_{55}^{\tau \tau^{\prime}}\left(\vec{n}-\vec{n}^{\prime}\right)$ for $l=5$ by a factor $1.5-5$ smaller than the corresponding coefficients of quadrupolar interactions calculated with SAF's $S_{2}^{E_{g}, 3}$ and $S_{2}^{E_{g}, 4}$. We take this fact as an indication that rotations of the $\mathrm{C}_{70}$ molecule about its fivefold axis still remain allowed. A freezing in of molecular orientations about the fivefold axis is not required for the low-symmetry phase which turns out to have a monoclinic structure (see below).

In the following we will only retain contributions from SAF's $S_{2}^{E_{g}, 3}$ and $S_{2}^{E_{g}, 4}$ to the rotation-rotation interaction $V^{\mathrm{RR}}$, expression (2.18). We simplify the notations by writing $S_{2}^{\alpha}$, where $\alpha=1$ stands for $\left(E_{g}, 3\right)$ and $\alpha=2$ for $\left(E_{g}, 4\right)$. We then study the eigenvalues of the $2 \times 2$ matrix $\mathcal{J}_{22}(\vec{q})$ with elements $\mathcal{J}_{22}^{\alpha \beta}(\vec{q})$ as a function of $\vec{q}$ in the Brillouin zone of the rhombohedral lattice. The largest negative eigenvalue is found at the $F$ point of the Brillouin zone and we will denote this eigenvalue by $J_{F}$ and the corresponding eigenvector by $\vec{e}_{F}$ with elements $e_{F}\left(\alpha \mid \vec{q}_{F}\right)$ :

$$
\mathcal{J}_{22}\left(\vec{q}_{F}\right) \vec{e}_{F}=J_{F} \vec{e}_{F} .
$$

We obtain $J_{F}=-36580 \mathrm{~K}$, the contributions from the different interaction centers are listed in Table II. There are six points in Brillouin zone giving the same eigenvalue $J_{F}$. They are connected with the $F$ point by symmetry operations of $C_{3}, C_{3}^{2}$, and $I$ (inversion) of the point group $D_{3 d}$ :

$$
\begin{gathered}
F: \quad \pm\left(\frac{\pi}{\sqrt{3} a},-\frac{\pi}{3 a}, \frac{2 \pi}{3 c}\right) ; \\
F^{\prime}: \quad \pm\left(0, \frac{2 \pi}{3 a}, \frac{2 \pi}{3 c}\right) ; \\
F^{\prime \prime}: \quad \pm\left(-\frac{\pi}{\sqrt{3} a},-\frac{\pi}{3 a}, \frac{2 \pi}{3 c}\right) .
\end{gathered}
$$

Here $a=5.846 \AA$ and $c=9.283 \AA$ are lattice constants of the rhombohedral lattice ${ }^{3}$ (see Fig. 2). The normalized eigenvectors are

$$
\vec{e}_{F}=\left[\begin{array}{c}
\frac{\sqrt{3}}{2} \\
-\frac{1}{2}
\end{array}\right] ; \quad \vec{e}_{F^{\prime}}=\left[\begin{array}{l}
0 \\
1
\end{array}\right] ; \quad \vec{e}_{F^{\prime \prime}}=\left[\begin{array}{c}
\frac{\sqrt{3}}{2} \\
\frac{1}{2}
\end{array}\right],
$$

and the corresponding normal coordinates of orientations are given by

$$
Q_{2}\left(\vec{q}_{p}\right)=\sum_{\alpha=1}^{2} e_{p}\left(\alpha \mid \vec{q}_{p}\right) \mathcal{U}_{2}^{\alpha}\left(\vec{q}_{p}\right)
$$

where the subscript $p$ stands for $F, F^{\prime}, F^{\prime \prime}$. The six points in the Brillouin zone correspond to physically equivalent states (domains). Since $\vec{e}_{F}$ is particularly simple, we will retain hereafter $Q_{2}\left(\vec{q}_{F^{\prime}}\right)$ as the order parameter variable. The condensation of this order parameter leads to a monoclinic structure. The symmetry lowering can be represented by the following diagram:

$$
R \overline{3} m: \quad\left[\vec{q}_{F^{\prime}} ; Q_{2}^{e}\left(\vec{q}_{F^{\prime}}\right)=N^{1 / 2} \eta \neq 0\right] \rightarrow P_{2_{1}} / m
$$

Here the index $e$ stands for a thermal expectation value, $\eta$ is the order-parameter amplitude. In real space we have

$$
\begin{gathered}
\left\langle U_{2}^{E_{g}, 4}(\vec{n})\right\rangle=\eta \cos \left(\vec{q}_{F^{\prime}} \cdot \vec{X}(\vec{n})\right), \\
\eta=\sqrt{\frac{4 \pi}{5}}\left\langle Y_{2}^{2, s}(\vec{n})\right\rangle,
\end{gathered}
$$

where the rotator function $U_{2}^{E_{g}, 4}$ is given by Eq. (A4). As follows from Eq. (3.7a), $\left\langle U_{2}^{E_{g}, 4}(\vec{n})\right\rangle$ changes from $+\eta$ to $-\eta$ for neighboring sites. Taking into account the angular dependence of $Y_{2}^{2, s}(\vec{n})$, we show in Fig. 3 the breaking of rhombohedral symmetry and formation of a new unit cell in the monoclinic phase. There are six molecules per unit cell.

\section{B. Crystal field $V^{R}$}

In order to study more quantitatively the phase transition $R \overline{3} m \rightarrow P_{2_{1}} / m$, we need to know the crystal field $V^{R}$ in the rhombohedral phase. Starting from the interaction Eq. (3.1) with parameters specified in Table I, we have calculated the coefficients $w_{l}^{A_{1 g}, \rho}$ by means of Eqs. (2.14) and (2.15), using the SAF's $S_{l}^{A_{1 g}, \rho}$ of Appendix A. The crystal field resulting from the leading coefficient, $w_{2}^{A_{1 g}, 1}=-26464 \mathrm{~K}$, is enormously strong. Such a strong crystal field prevents any tilting of the long molecular axis away from the threefold trigonal axis and hence precludes a phase transition to a lowsymmetry structure. The reason for the strong crystal field is an artefact of our calculation. For elongated molecules like $\mathrm{C}_{70}$ on a rigid lattice, the treatment of the 12 neighbors of a central molecule as spheres (see Sec. II) is a too crude approximation. Indeed by averaging the positions of interaction centers over spheres with radii corresponding to their distances from the center of the molecule, the repulsive forces between neighboring molecules on a rigid lattice are overestimated. We observe that molecular-dynamics simulations ${ }^{14}$ are not subject to these restrictions. Going one step beyond this approximation, we replace the neighboring molecules by quadrupoles $S_{2}^{A_{1 g}, 1}=Y_{2}^{0}$ and calculate the coefficients $J_{l 2}^{A_{1 g}, \rho ; A_{1 g}, 1}\left(\vec{n}-\vec{n}^{\prime}\right)$ by means of Eqs. (2.11) and (2.12). We obtain for the corresponding crystal field at site $\vec{n}$ : 


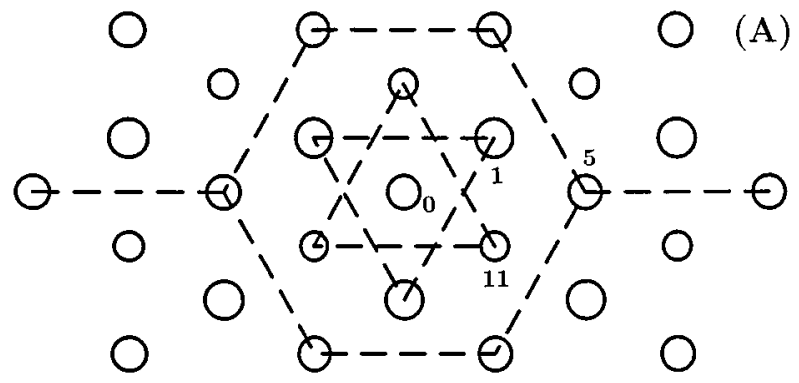

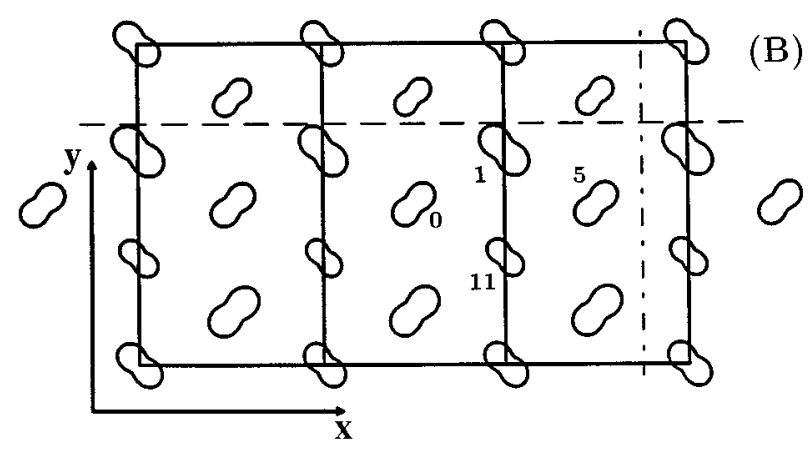

FIG. 3. The transition from rhombohedral $(A)$ to monoclinic $(B)$ lattice and formation of the monoclinic primitive unit cell. Projection on the $(x, y)$ plane; labels 1,11 , and 5 are shown for comparison with Fig. 2. The drawn symbols indicate the main transformation properties of the corresponding orientational crystal field. Circles $(A)$ represent the equivalent crystal field for every site in the rhombohedral phase. In the monoclinic phase $(B)$ the breaking of $D_{3 d}$ site symmetry is shown by drawing positive lobes of $U_{2}^{E, 4}$ $\propto Y_{2}^{2, s}$ function at different lattice sites. Solid lines confine the monoclinic unit cells $(B)$. Different crystal planes are represented by different size of symbols. The screw axis (dashed line in the base plane) and glide reflection plane (dotted and dashed line) of symmetry group $P 2_{1} / \mathrm{m}$ are shown for the monoclinic phase $(B)$.

$$
\begin{aligned}
\sum_{n^{\prime}}^{12} V_{l 2}^{A_{1 g}, \rho ; A_{1 g}, 1}\left(\vec{n}-\vec{n}^{\prime}\right)= & \sum_{n^{\prime}} J_{l 2}^{A_{1 g}, \rho ; A_{1 g}, 1}\left(\vec{n}-\vec{n}^{\prime}\right) \\
& \times U_{l}^{A_{1 g}, \rho}(\vec{n}) U_{2}^{A_{1 g}, 1}\left(\vec{n}^{\prime}\right)
\end{aligned}
$$

In the mean-field approximation we replace $U_{2}^{A_{1 g}, 1}\left(\vec{n}^{\prime}\right)$ by its crystal-field average and rewrite expression (3.8) as

$$
\begin{aligned}
\sum_{\vec{n}^{\prime}} & J_{l 2}^{A_{1 g}, \rho ; A_{1 g}, 1}\left(\vec{n}-\vec{n}^{\prime}\right) U_{l}^{A_{1 g}, \rho}(\vec{n})\left\langle U_{2}^{A_{1 g}, 1}\left(\vec{n}^{\prime}\right)\right\rangle_{\mathrm{CF}} \\
& =\widetilde{w}_{l}^{A_{1 g}, \rho} U_{l}^{A_{1 g}, \rho}(\vec{n})
\end{aligned}
$$

where

$$
\widetilde{w}_{l}^{A_{1 g}, \rho}=\sum_{\vec{n}^{\prime}} J_{l 2}^{A_{1 g}, \rho ; A_{1 g}, 1}\left(\vec{n}-\vec{n}^{\prime}\right)\left\langle U_{2}^{A_{1 g}, 1}\left(\vec{n}^{\prime}\right)\right\rangle_{\mathrm{CF}}
$$

We observe that \langle\rangle$_{\mathrm{CF}}$ is independent of $\vec{n}^{\prime}$ and that $w_{l \mathrm{CF}}^{A_{1 g}, \rho}$ is independent of $\vec{n}$ (equivalence of rhombohedral lattice sites). Including the contributions Eq. (3.9), we replace the crystalfield expression (2.13) by

$$
V_{\mathrm{CF}}=\sum_{\vec{n}} \sum_{l} \sum_{\rho} w_{l \mathrm{CF}}^{A_{1 g}, \rho} U_{l}^{A_{1 g}, \rho}(\vec{n})
$$

where

$$
w_{l \mathrm{CF}}^{A_{1 g}, \rho}=w_{l}^{A_{1 g}, \rho}+\widetilde{w}_{l}^{A_{1 g}, \rho} .
$$

The thermal average in Eq. (3.10) is found by solving selfconsistently the equation

$$
\begin{aligned}
\left\langle U_{2}^{A_{1 g}, 1}\right\rangle_{\mathrm{CF}} & =\frac{1}{\mathcal{Z}} \int d \omega \exp \left[-V_{\mathrm{CF}}(\omega) / T\right] U_{2}^{A_{1 g}, 1}(\omega), \\
\mathcal{Z} & =\int d \omega \exp \left[-V_{\mathrm{CF}}(\omega) / T\right],
\end{aligned}
$$

where $d \omega=\sin \beta d \beta d \alpha, \omega=(\beta, \alpha)$ being the polar angles of the long molecular axis. Calculating $\widetilde{w}_{2}^{A_{1 g}, 1}$ by means of Eqs. (3.10), (3.13a), and (3.13b), we obtain a positive value, $20854 \mathrm{~K}$, for $T=285 \mathrm{~K}$. Hence it follows from Eq. (3.12) that the leading crystal-field coefficient is strongly reduced, $w_{2 \mathrm{CF}}^{A_{1 g}, 1}=-5610 \mathrm{~K}$ instead of $w_{2}^{A_{1 g}, 1}$. This reduction of the crystal field means that the system will become more susceptible to orientational fluctuations which lower the rhombohedral symmetry and lead to a monoclinic phase of space group $P_{2_{1}} / m$. The crystal-field coefficients are quoted in Table III. They are slightly temperature dependent. As an indication the $T$ dependence of $\left\langle U_{2}^{A_{1 g}, 1}\right\rangle_{\mathrm{CF}}$ in the rhombohedral phase is shown in Fig. 4. The nature of the average $\left\langle U_{2}^{A_{1 g}, 1}\right\rangle_{\mathrm{CF}} \equiv \zeta$ is also discussed in Sec. V.

As an additional mechanism which modifies the crystal field, we have taken into account the orientational selfinteraction $V^{s}$ of the $\mathrm{C}_{70}$ molecule with the lattice deformations. Self-interactions are a consequence of bilinear rotation-translation (RT) coupling. These effects have been studied thoroughly for the fcc phases of KCN (Ref.27) and of $\mathrm{C}_{70}$ (Ref.12). For the present work, we have extended the method to the rhombohedral phase of $\mathrm{C}_{70}$. Without giving the details of the calculations, we only mention that the decomposition of the square of the rotator functions, $\left(U_{2}^{\alpha}\right)^{2}$, where $\alpha=\left(E_{g}, 3\right)$ and $\left(E_{g}, 4\right)$, contains the functions $U_{l}^{A_{1 g}, 1}$

TABLE III. Crystal-field coefficients, in $\mathrm{K}\left(T_{1}=285 \mathrm{~K}\right)$.

\begin{tabular}{lcccccccc}
\hline \hline$(l, \rho)$ & $(2,1)$ & $(4,1)$ & $(6,1)$ & $(8,1)$ & $(4,2)$ & $(6,2)$ & $(6,3)$ & $(8,3)$ \\
$w_{l}^{A_{1 g}, \rho}$ & -26464 & 1712 & 2109 & -1463 & 66 & -294 & -4071 & 2677 \\
$\widetilde{w}_{l}^{A_{1 g}, \rho}\left(T_{1}\right)$ & 20854 & -1019 & -1210 & 1192 & 63 & -257 & 2660 & -1708 \\
$w_{l \mathrm{~A}}^{A_{1 g}, \rho}\left(T_{1}\right)$ & -5610 & 693 & 899 & -271 & 130 & -551 & -1412 & 969 \\
$w_{l \mathrm{~A} F}^{A_{1 g}, \rho}\left(T_{1}, \mathrm{RT}\right)$ & -5147 & 554 & 899 & -271 & 130 & -551 & -1412 & 969 \\
\hline \hline
\end{tabular}




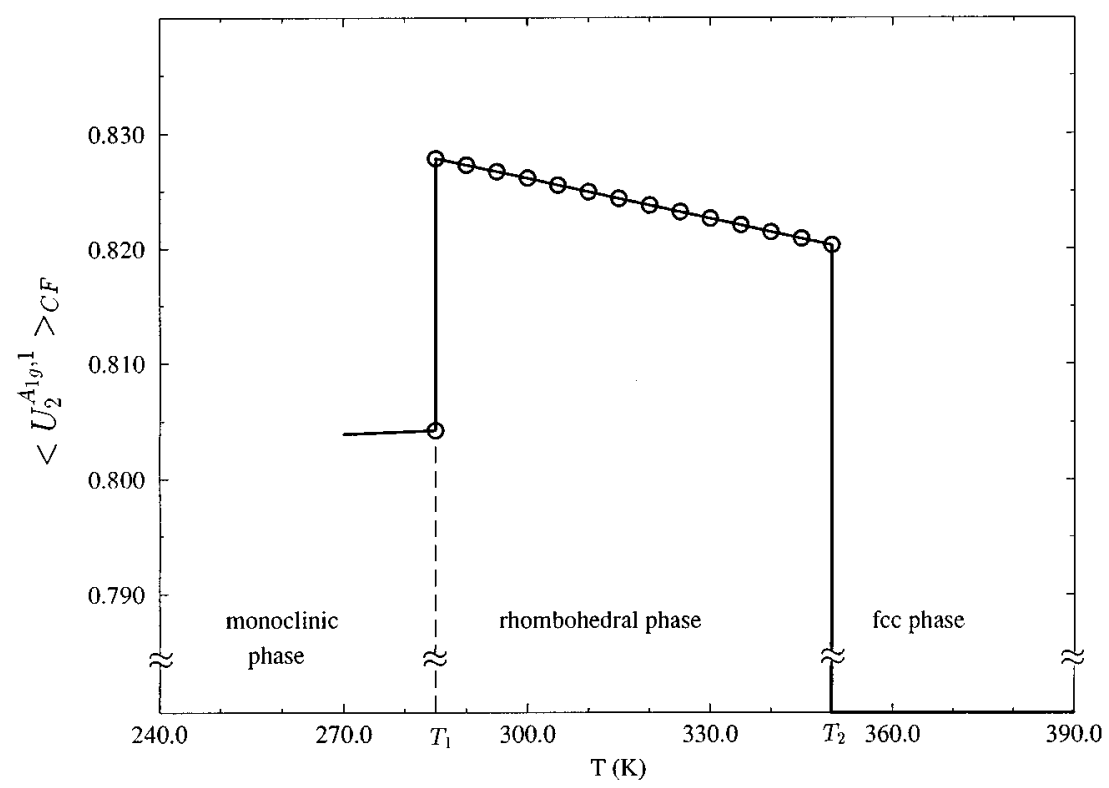

FIG. 4. Temperature dependence of $\left\langle U_{2}^{A_{1}, 1}\right\rangle_{\mathrm{CF}}$ in the rhombohedral phase. It corresponds to a change of the orientational crystal field. With respect to the phase transition to the monoclinic structure $\left\langle U_{2}^{A_{1}, 1}\right\rangle_{\mathrm{CF}}$ acts as a secondary order parameter which exhibits a jump to a smaller value at the transition, as is also shown here.

$l=2,4$. The self-interaction then leads to a modification of the crystal-field coefficients $w_{2 \mathrm{CF}}^{A_{1 g}, 1}$ and $w_{4 \mathrm{CF}}^{A_{1 g}, 1}$ These modified coefficients are quoted as $w_{l \mathrm{CF}}^{A_{1 g}, \rho}\left(T_{1}, \mathrm{RT}\right)$ in Table III.

In Fig. 5 we show a three-dimensional plot of the crystalfield, obtained from expression (3.11) with the crystal field coefficients given in the last line of Table III. Our calculations indicate that there are three shallow degenerate minima around the $z$ axis with $\triangle V / V_{\mathrm{CF}}(\Theta=0) \approx 0.01$ at angles $\Theta=0.38$ and $\phi=\pi / 2,7 \pi / 6,11 \pi / 6$, where $\Omega=(\Theta, \phi)$ are the polar angles of the long axis of the molecule. Since the orientational probability distribution is given by $P(\Omega)$ $\propto \exp \left[-V_{\mathrm{CF}}(\Omega) / T\right]$ we conclude that there is a precessional motion of the long axis around the $\vec{c}$ direction in the rhombohedral phase. These results agree with moleculardynamics simulations. ${ }^{14}$

If for the time being we assume that the phase transition is of the second order, we find by Landau theory ${ }^{28,29}$ that the transition temperature $T_{c}$ is given by the solution of the implicit equation

$$
T_{c}+J_{F} x^{(2)}\left(T_{c}\right)=0
$$

Here $x^{(2)}(T)$ is the single-particle expectation value

$$
x^{(2)}(T)=\left\langle\left(\mathcal{U}_{2}^{E_{g}, 4}(\vec{n})\right)^{2}\right\rangle_{\mathrm{CF}} .
$$

We find $T_{c}=271 \mathrm{~K}$.

\section{FREE ENERGY}

In order to investigate more closely the thermal changes that accompany the phase transitions $R \overline{3} m \rightarrow P_{2_{1}} / m$, we have derived the free energy in the rhombohedral phase. Restricting ourselves to the rotational degrees of freedom, and using the method of Refs. 30 and 31, we find for the Landau expansion $^{28}$ of the free energy per molecule

$$
F=F_{0}+F^{(2)}+F^{(4)}+F^{(6)}=F_{0}+A \eta^{2}+B \eta^{4}+C \eta^{6} .
$$

Here $\eta$ stands for the instantaneous expectation value of the orientational order-parameter variable $U_{2}^{E_{g}, 4}$ [see Eqs. (3.7a), (3.7b)]. Using symmetry arguments we can show that the expansion (4.1) contains only even powers in $\eta . F_{0}$ is the single-particle orientational free energy, and the coefficients of the expansion are obtained as

$$
\begin{gathered}
A=\frac{1}{2}\left\{\frac{T}{x^{(2)}}+J_{F}\right\}, \\
B=\frac{T}{24\left[x^{(2)}\right]^{2}}\left\{3-\frac{x^{(4)}}{\left[x^{(2)}\right]^{2}}\right\}, \\
C=\frac{T}{6 x^{(2)}}\left\{\frac{1}{2\left[x^{(2)}\right]^{2}}-\frac{3 x^{(4)}}{8\left[x^{(2)}\right]^{4}}+\frac{\left[x^{(4)}\right]^{2}}{12\left[x^{(2)}\right]^{6}}-\frac{x^{(6)}}{120\left[x^{(2)}\right]^{5}}\right\},
\end{gathered}
$$

where

$$
\begin{aligned}
& x^{(2)}=\left\langle\left[U_{2}^{E, 2, s}\right]^{2}\right\rangle_{\mathrm{CF}} ; \\
& x^{(4)}=\left\langle\left[U_{2}^{E, 2, s}\right]^{4}\right\rangle_{\mathrm{CF}} ; \\
& x^{(6)}=\left\langle\left[U_{2}^{E, 2, s}\right]^{6}\right\rangle_{\mathrm{CF}} .
\end{aligned}
$$

With the crystal-field coefficients from Table IV we obtain $\quad x^{(2)}=7.39 \times 10^{-3}, \quad x^{(4)}=2.24 \times 10^{-4}, \quad x^{(6)}$ $=1.18 \times 10^{-5}, B=-2.31 \times 10^{5} \mathrm{~K}$, and $C=1.38 \times 10^{7} \mathrm{~K}$. Since the coefficient $B$ of the fourth-order term is negative, the phase transition is of first order. ${ }^{28}$ The discontinuity of the order parameter at the phase transition is given by

$$
\eta_{1}= \pm \sqrt{-\frac{B}{2 C}}= \pm 0.092
$$

while the transition temperature, obtained from the solution of $B^{2}-4 A C$, is $T_{1}=T_{c}+\triangle T=285 \mathrm{~K}$ (notice $T_{c}=271 \mathrm{~K}$ ). The latent heat per molecule emitted at $T_{1}$ when the system transforms from the rhombohedral to the monoclinic phase is given by 


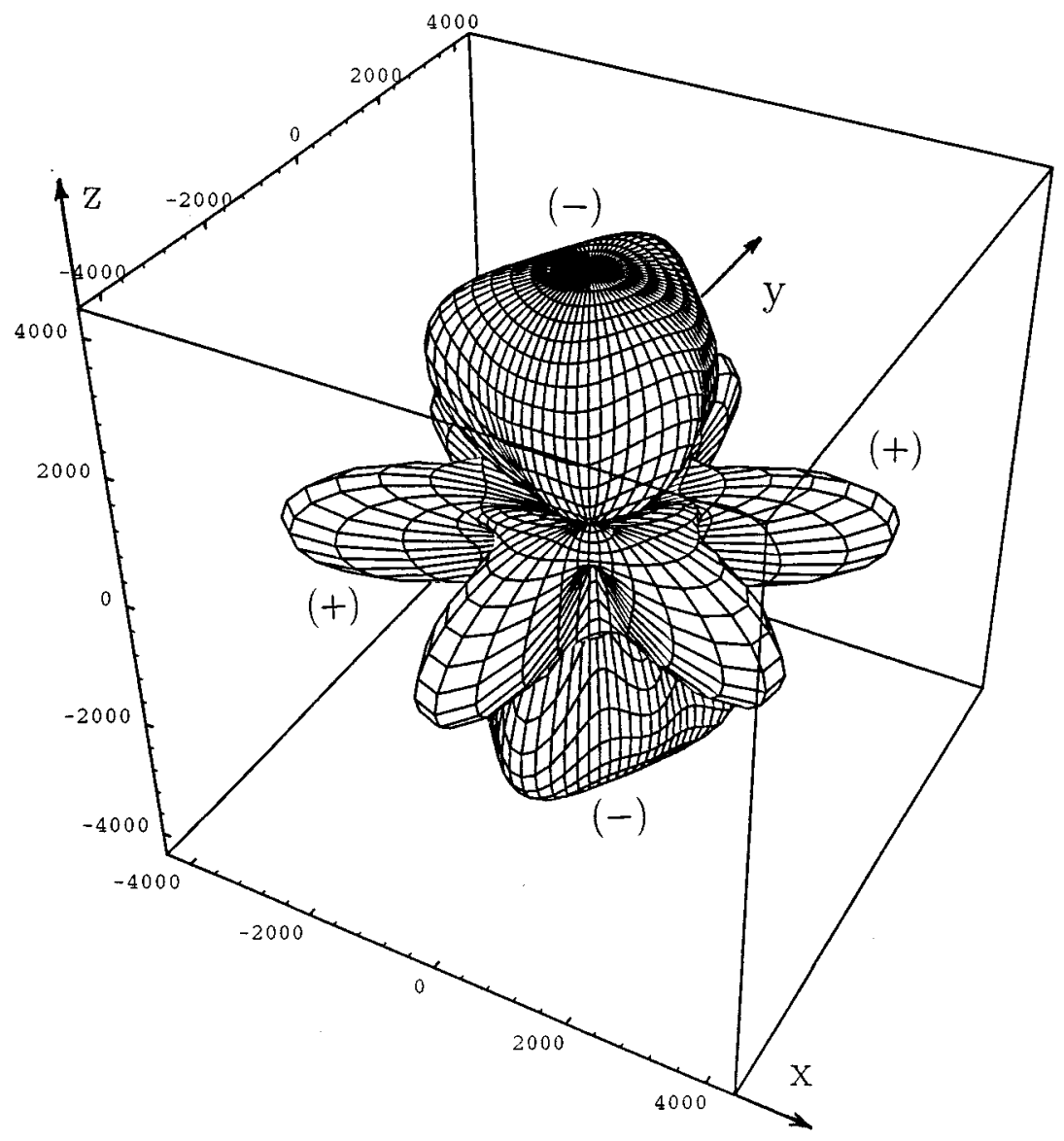

FIG. 5. The orientational crystal field (in K) of the rhombohedral phase, slightly above $T_{1}=285 \mathrm{~K}$. The polar regions around the $z$ axis marked by $(-)$ have negative sign (attraction), the equatorial region marked by $(+)$ has positive sign (repulsion).

$$
\begin{aligned}
\triangle H & =T_{1}\left(S^{>}-S^{<}\right)=-T_{1}\left(-\frac{\partial F_{0}(T)}{\partial T}+\frac{\partial F(T)}{\partial T}\right) \mid T_{1} \\
& =\frac{T_{1}}{x^{(2)}} \eta_{1}{ }^{2}+B\left(T_{1}\right) \eta_{1}{ }^{4}+C\left(T_{1}\right) \eta_{1}{ }^{6}
\end{aligned}
$$

TABLE IV. Crystal-field parameters $w_{l}^{A_{1 g}}$ in $\mathrm{K}$, in the fcc phase calculated for the present potential and for the potential of Ref. 12. Critical temperature $T_{c}$ in $\mathrm{K}$, for the transition $F m \overline{3} m \rightarrow R \overline{3} m$. We give two columns for the present potential, showing that $T_{c}^{\prime}$ and the first-order transition temperature $\left(T_{2}=T_{c}^{\prime}+\triangle T\right)$ depend strongly on the actual value of the elastic constant $c_{44}^{0}$ (The other elastic constants are kept the same as in Ref. 12, that is $c_{11}^{0}=2235$ and

\begin{tabular}{|c|c|c|c|}
\hline & \multicolumn{2}{|c|}{ Present } & Ref.12 \\
\hline$a$ & \multicolumn{2}{|c|}{14.892} & 15.0 \\
\hline$w_{4}$ & \multicolumn{2}{|c|}{-348.4} & -535.4 \\
\hline$w_{6}$ & \multicolumn{2}{|c|}{2085.3} & 1794.6 \\
\hline$w_{8}$ & \multicolumn{2}{|c|}{-1091.9} & -1353.7 \\
\hline$c_{44}^{0}$ & 875 & 920 & 875 \\
\hline$T_{c}^{\prime}$ & 504 & 346 & 457 \\
\hline
\end{tabular}
$c_{12}^{0}=950$, in $\mathrm{K} / \AA$.) $a$ is fcc lattice constant, in $\AA^{3}$.
Here $S^{>}$and $S^{<}$denote the entropy just above and below $T_{1}$. Our calculation yields $\triangle H=2.61 \mathrm{~kJ} \mathrm{~mol}^{-1}$. The value is comparable with experimentally found ${ }^{1,3} 3.5 \pm 0.5 \mathrm{~kJ}$ $\mathrm{mol}^{-1}, 3.4 \pm 0.2 \mathrm{~kJ} \mathrm{~mol}^{-1}$, and $2.3 \pm 0.4 \mathrm{~kJ} \mathrm{~mol}^{-1}$ (Ref. 1, cited in Ref. 14). It is also consistent with results of molecular-dynamics simulations ${ }^{14}$ giving $\triangle H=2.5 \pm 0.3 \mathrm{~kJ}$ $\mathrm{mol}^{-1}$.

Although we have treated here the transition from the rhombohedral to the monoclinic phase, we want to mention that the present potential with parameters from Table I also describes the transition from the cubic to the rhombohedral phase. A comparison of the calculated values of the crystalfield coefficients and of $T_{c}$ with the values of Ref.12 is given in Table IV. We observe that a main advantage of the present potential is that the reduction of the strength $C^{(1)}$ of the repulsive potentials which involve intermediate bond interactions leads to a reduction of the crystal field in the monoclinic phase. This is illustrated by the values for $w_{2 \mathrm{CF}}^{A_{1 g}, 1}$ given in Table $\mathrm{V}$.

\section{LATTICE DISTORTIONS}

The orientational ordering at the transition and at lower temperature in the monoclinic phase is accompanied by a 
TABLE V. Partial contribution of $C, D, I$ centers to the leading crystal-field coefficient $w_{2 \mathrm{CF}}^{A_{1 g}, 1}$ in $\mathrm{K}$.

\begin{tabular}{lccccccc}
\hline \hline & $C, C$ & $C, D$ & $D, D$ & $I, C$ & $I, D$ & $I, I$ & Total \\
\hline$w_{2 \mathrm{CF}}^{A_{1,1}, 1}\left(T_{1}=285 \mathrm{~K}\right)$ & -2115 & -2750 & 151 & -419 & -497 & 20 & -5610 \\
\hline \hline
\end{tabular}

distortion of the crystal lattice occupied by the center-ofmass positions of the $\mathrm{C}_{70}$ molecules. Here we want to investigate these effects which are a consequence of rotationrotation-translation (RRT) coupling. ${ }^{27,32} \mathrm{We}$ consider the orientational interaction $V^{\mathrm{RR}}$, Eq. (2.10), on a deformable lattice. The centers-of-mass positions are then given [compare with Eq. (2.8)] by

$$
\vec{R}(\vec{n}, \lambda, \nu)=\vec{X}(\vec{n})+\vec{u}(\vec{n}),
$$

where $\vec{u}(\vec{n})$ are the lattice displacements. Expanding $V^{\mathrm{RR}}$ in terms of $\vec{u}$, we obtain as a linear correction to the rigid lattice term Eq. (2.10)

$$
V^{\mathrm{RRT}}=\frac{1}{2} \sum_{n n^{\prime}} \mathcal{V}_{i}^{\alpha \beta}\left(\vec{n}-\vec{n}^{\prime}\right) U^{\alpha}(\vec{n}) U^{\beta}\left(\vec{n}^{\prime}\right)\left[u_{i}(\vec{n})-u_{i}\left(\vec{n}^{\prime}\right)\right],
$$

where $\alpha$ stands for $(\tau, l)$ and where $\mathcal{V}_{i}^{\alpha \beta}$ is derivative of $J^{\alpha \beta}\left(\vec{n}-\overrightarrow{n^{\prime}}\right)$ with respect to $X_{i}(\vec{n})$. Transforming to Fourier space we find

$$
V^{\mathrm{RRT}}=\frac{1}{2} \sum_{p q} \mathcal{V}_{i}^{\alpha \beta}(\vec{q}, \vec{p}) \mathcal{U}^{\alpha}(-\vec{q}-\vec{p}) \mathcal{U}^{\beta}(\vec{p}) u_{i}(\vec{q}),
$$

where

$$
\begin{aligned}
\mathcal{V}_{i}^{\alpha \beta}(\vec{q}, \vec{p})= & (N m)^{-1 / 2} \sum_{\vec{\kappa}} \mathcal{V}_{i}^{\alpha \beta}(\vec{\kappa}) \cos \left[\left(\vec{p}+\frac{\vec{q}}{2}\right) \cdot \vec{X}(\vec{\kappa})\right] \\
& \times \sin \left[\frac{\vec{q} \cdot \vec{X}(\vec{\kappa})}{2}\right] .
\end{aligned}
$$

Here we have defined $\vec{\kappa}=\vec{n}^{\prime}-\vec{n}$. The vector $\vec{X}(\vec{\kappa})=\vec{X}\left(\vec{n}^{\prime}-\vec{n}\right)$ denotes the positions of the 12 nearest neighbors with respect to the central molecule $\vec{n}$ in the rhombohedral lattice. The Fourier transformed displacements are given by

$$
\vec{u}(\vec{q})=\sqrt{\frac{m}{N}} \sum_{\vec{n}} \vec{u}(\vec{n}) e^{-i \vec{q} \cdot \vec{X}(\vec{n})},
$$

where $m$ is the mass of the $\mathrm{C}_{70}$ molecule. We consider expression (5.3a) in the long wave limit $\vec{q} \rightarrow 0$ for the lattice displacements and define the homogeneous strains by

$$
\frac{i q_{j} u_{i}(\vec{q})}{\sqrt{m N}}=\epsilon_{i j} \delta(\vec{q}) .
$$

Replacing the rotator functions $\mathcal{U}^{\alpha}( \pm \vec{p})$ in expression (5.3a) by the orientational order parameter

$$
\left\langle\mathcal{U}_{2}^{E_{g}, 4}(\vec{p})\right\rangle=\eta \sqrt{N}\left(\delta_{\vec{p}, \vec{q}_{F^{\prime}}}+\delta_{\vec{p},-\vec{q}_{F^{\prime}}}\right) / 2,
$$

we obtain for the contribution of $V^{\mathrm{RRT}}$ to the free energy per molecule

$$
V^{\mathrm{RRT}}[\eta, \epsilon]=\eta^{2}\left(\Lambda_{x x} \epsilon_{x x}+\Lambda_{y y} \epsilon_{y y}+\Lambda_{z z} \epsilon_{z z}+\Lambda_{y z} \epsilon_{y z}\right) V_{c},
$$

where $V_{c}$ is the volume per unit cell. Here we have defined the coefficients

$$
\Lambda_{i j}=\frac{1}{2} \sum_{\vec{\kappa}} \mathcal{V}_{i}^{\alpha \alpha}(\vec{\kappa}) \cos \left[\vec{q}_{F^{\prime}} \cdot \vec{X}(\vec{\kappa})\right] \vec{X}_{j}(\vec{\kappa}),
$$

where $\alpha=E_{g}, 4$. Numerical calculations for these quantities with the potential parameters of Table I yield $\Lambda_{x x}=197$, $\Lambda_{y y}=510, \Lambda_{z z}=2.84, \Lambda_{y z}=-0.205$, in units $\mathrm{K} / \AA^{3}$.

We add to the free energy an elastic term of the rhombohedral crystal

$$
\begin{aligned}
F^{\mathrm{TT}}[\epsilon]= & \left\{\frac{1}{2} c_{33}^{0} \epsilon_{z z}^{2}+\frac{1}{2} c_{11}^{0}\left(\epsilon_{x x}^{2}+\epsilon_{y y}^{2}\right)+c_{12}^{0} \epsilon_{x x} \epsilon_{y y}\right. \\
& +\left(c_{11}^{0}-c_{12}^{0}\right) \epsilon_{x y}^{2}+c_{13}^{0}\left(\epsilon_{x x}+\epsilon_{y y}\right) \epsilon_{z z} \\
& +2 c_{44}^{0}\left(\epsilon_{x z}^{2}+\epsilon_{y z}^{2}\right)+2 c_{14}^{0}\left[\left(\epsilon_{x x}-\epsilon_{y y}\right) \epsilon_{y z}\right. \\
& \left.\left.+2 \epsilon_{x y} \epsilon_{x z}\right]\right\} V_{c} .
\end{aligned}
$$

Here $c_{i j}^{0}$ are the bare elastic constants in Voigt's notation. The bare elastic constants $c_{i j}^{0}$ are the elastic constants in absence of any coupling of the lattice displacements to molecular rotations. ${ }^{13}$ The last expression is written for a coordinate system which differs from the one used in Ref.34 by permutation of $x$ and $y$ axes. Since we do not know so far about experimental data on the elastic constants of $\mathrm{C}_{70}$ in the rhombohedral phase, we have made numerical estimates by performing a model calculation of the $c_{i j}^{0}$. Details of the calculations and relations between elastic constants and Lamé coefficients used by Landau and Lifshitz are given in Appendix B.

The form of expression (5.7) for $F^{\mathrm{RRT}}[\eta, \epsilon]$ implies that only distortions with $\epsilon_{x x} \neq 0, \epsilon_{y y} \neq 0, \epsilon_{z z} \neq 0, \epsilon_{y z} \neq 0$ occur in the monoclinic phase, while the other components of the strain tensor will be zero $\left(\epsilon_{x y}=0, \epsilon_{x z}=0\right)$. In principle, positive values of $\Lambda_{i i}$, where $i i=x x, y y, z z$ lead to negative values of $\epsilon_{x x}, \epsilon_{y y}, \epsilon_{z z}$, thus giving a tendency to contraction of the monoclinic unit cell in all directions. However, in the elastic energy $F^{\mathrm{TT}}[\epsilon]$ there exists a coupling term, $c_{13}^{0}\left(\epsilon_{x x}+\epsilon_{y y}\right) \epsilon_{z z}$, which causes an elongation along the $z$ axis. As a result, the sign of the distortion $\epsilon_{z z}$ depends on the numerical values of the elastic constants.

The remaining nonzero component $\epsilon_{y z}$ of strain tensor gives rise to a distortion which is not reduced to contraction or elongation in the $x, y, z$ directions, see Fig. 6 . It brings a shear deformation of the monoclinic unit cell characterized by the monoclinic angle $\beta$ :

$$
\cos \beta=\left|2 \epsilon_{y z}\right| .
$$




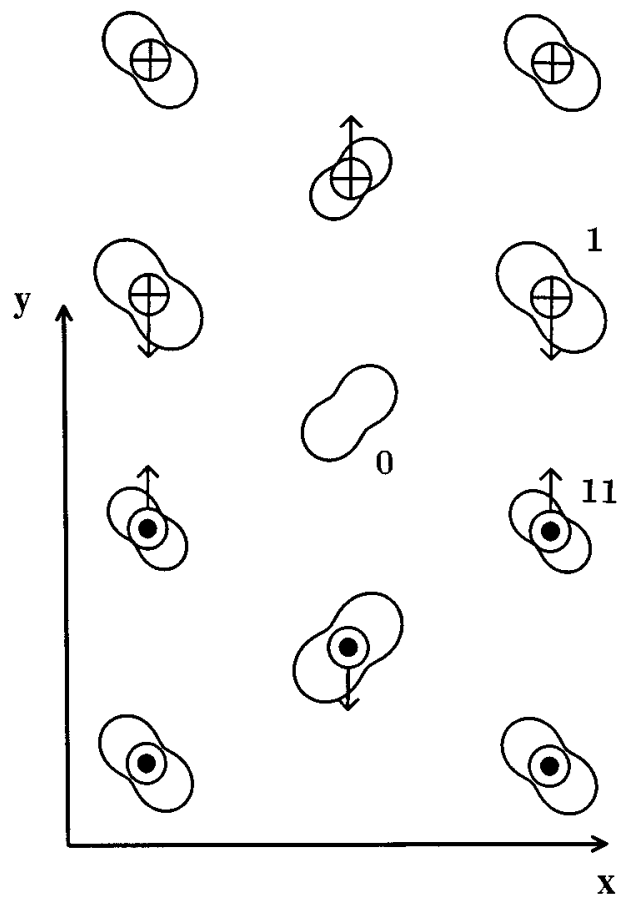

FIG. 6. $\epsilon_{y z}<0$ distortion of the monoclinic unit cell. Symbol $\oplus$ denotes displacement behind the plane of the picture, $\odot$ denotes above the plane in the direction perpendicular to the plane. Displacements on the $(x y)$ plane of the picture are shown by arrows. The distortion preserves the glide reflection plane and screw axis of $P 2_{1} / m$ site symmetry.

We now give numerical estimates of the strains. The lattice distortion corresponding to an orientational order $\eta$ in the monoclinic phase is determined by the minimialization conditions of the free energy:

$$
\frac{\partial\left\{F^{\mathrm{RRT}}[\eta, \epsilon]+F^{T T}[\epsilon]\right\}}{\partial \epsilon_{i j}}=0,
$$

where $i j=x x, y y, z z$ and $y z$. Explicitly we obtain

$$
\begin{gathered}
c_{11}^{0} \epsilon_{x x}+c_{12}^{0} \epsilon_{y y}+c_{13}^{0} \epsilon_{z z}+2 c_{14}^{0} \epsilon_{y z}+\mathcal{L}_{x x}=0, \\
c_{11}^{0} \epsilon_{x x}+c_{12}^{0} \epsilon_{y y}+c_{13}^{0} \epsilon_{z z}-2 c_{14}^{0} \epsilon_{y z}+\mathcal{L}_{y y}=0, \\
c_{33}^{0} \epsilon_{z z}+c_{13}^{0}\left(\epsilon_{x x}+\epsilon_{y y}\right)+\mathcal{L}_{z z}=0, \\
4 c_{44}^{0} \epsilon_{y z}+2 c_{14}^{0}\left(\epsilon_{x x}-\epsilon_{y y}\right)+\mathcal{L}_{y z}=0,
\end{gathered}
$$

where we have defined $\mathcal{L}_{i j}=\Lambda_{i j} \eta^{2}$. With our choice of elastic constants we have solved this system of equations for $\epsilon_{i j}$ and obtained

$$
\begin{gathered}
\boldsymbol{\epsilon}_{x x}=-0.0407 \eta^{2}, \quad \epsilon_{z z}=0.0320 \eta^{2}, \\
\epsilon_{y y}=-0.1598 \eta^{2}, \quad \epsilon_{y z}=-0.0205 \eta^{2} .
\end{gathered}
$$

At the phase transition, $\eta^{2} \approx 0.0085$. Therefore, for our choice of elastic constants (Appendix B) we have contractions in the $x, y$ direction and an elongation in the $z$ direction. Experimental data for $T=15 \mathrm{~K}$ indicate that there are contractions in all three directions ${ }^{3}$ with $\epsilon_{x x}$ $=-0.0195, \epsilon_{y y}=-0.00496, \epsilon_{z z}=-0.0028,\left|\epsilon_{y z}\right|=0.0045$.
Notice that, as we mentioned before, it is possible to obtain a negative value of $\epsilon_{z z}$ if a smaller value of the elastic constant $c_{13}^{0}<14.9 \mathrm{~K} / \AA^{3}$ is taken.

Although the calculated values of $\epsilon_{i j}$ are quantitatively still different from the experimental data at $15 \mathrm{~K}$, the theory correctly predicts which lattice deformations are affected by the transition from the rhombohedral to the monoclinic phase.

In order to improve the agreement with experiment, we have considered as an additional mechanism the coupling between orientational modes of rhombohedral symmetry $\left(A_{1 g}\right)$ and lattice strains. Initially, this coupling originates from corresponding rotation-translation (RT) interaction. However, it should be modified by taking into account a coupling between two SAF's $S_{2}^{A_{1 g}, 1}$ in a way which is similar to the modification of the crystal field described in Sec. III B. Such a modified rotation-translation (RT) coupling leads to a free-energy contribution per molecule

$$
F^{\mathrm{RT}}[\zeta, \epsilon]=\zeta\left[\alpha\left(\epsilon_{x x}+\epsilon_{y y}\right)-\beta \epsilon_{z z}\right] V_{c}
$$

in the rhombohedral phase. For the coupling parameters $\alpha$ and $\beta$ we find $130 \mathrm{~K} / \AA^{3}$ and $80 \mathrm{~K} / \AA^{3}$, respectively, and $\zeta=\left\langle U_{2}^{A_{1 g}, 1}\right\rangle$ is a thermal expectation value. Moreover, $\zeta$ can be regarded as the order parameter of the fcc $\rightarrow$ rhombohedral phase transition condensing at the transition temperature $T_{2} \approx 350 \mathrm{~K}$. Since in the disordered phase $\zeta=0$ and in the rhombohedral phase $\zeta>0$ (Fig. 4), it follows from Eqs. (5.9) and (5.14) that the fcc $\rightarrow$ rhombohedral phase transition is accompanied with a decrease of $\epsilon_{x x}$ and $\epsilon_{y y}$ and an increase of $\epsilon_{z z}$.

We can calculate values of $\zeta$ in the monoclinic phase if in Eqs. (3.13a), (3.13b) we consider the orientational molecular field

$$
V_{\mathrm{MF}}(\omega, \eta)=V_{\mathrm{CF}}(\omega)+J_{F} \eta U_{2}^{E_{g}, 4}(\omega),
$$

instead of the crystal field $V_{\mathrm{CF}}(\omega)$. If in Eq. (5.15) we take $\eta=\eta_{1}= \pm 0.092$ which corresponds to the value of the primary order parameter $\left\langle U_{2}^{E_{g},{ }^{4}}\right\rangle$ just below the temperature $T_{1}=285 \mathrm{~K}$ of the rhombohedral $\rightarrow$ monoclinic phase transition (see Sec. IV), we obtain $\zeta=0.804$. Thus at the transition from the rhombohedral to the monoclinic phase, the quantity $\zeta$ undergoes a discontinuous decrease $\triangle \zeta=-0.0236$ (see Fig. 4). The concomitant changes in $\epsilon_{x x}$ and $\epsilon_{y y}$ are positive $\left(\epsilon_{x x}=\epsilon_{y y}=3.426 \times 10^{-4}\right)$, while there is a negative change in $\epsilon_{z z}\left(\epsilon_{z z}=-2.782 \times 10^{-4}\right)$. In the monoclinic phase, the quantity $\zeta$ plays the role of a secondary order parameter which decreases with decreasing $T$. It is conceivable that the concomitant decrease of $\epsilon_{z z}$ then competes with the increase of $\epsilon_{z z}$ derived from expression (5.13) and ultimately leads to a negative value at low $T$ as is found by experiment. ${ }^{3}$

\section{CONCLUDING REMARKS}

Starting from a phenomenological model of microscopic interactions between neighboring $\mathrm{C}_{70}$ molecules, we have studied the orientation-dependent intermolecular potential in the rhombohedral phase. We find that the interactions between orientational modes of the manifold $l=2$ (quadrupoles) which transform as the irreducible representation $E_{g}$ 
of the rhombohedral site group $D_{3 d}$ drive the transition to a monoclinic phase. These interactions become attractive at the $F$ point of the Brillouin zone, the monoclinic phase is found to have space group $P_{2} / m$, in agreement with experiment $^{3,20}$ and computer simulations. ${ }^{21} \mathrm{We}$ have extensively studied the crystal field in the rhombohedral phase (see Figs. 4 and 5). In the rhombohedral phase, the molecule is quasifreely rotating about its long fivefold axis, the average orientation of the axis is along the rhombohedral $c$ direction (the [111] direction of the high temperature cubic phase). However, a precessional motion of the long axis about the rhombohedral $c$ axis is found, in agreement with molecular-dynamics simulations. ${ }^{14}$

The interactions involving orientational modes belonging to the manifold $l=5$ are weaker and we have no theoretical evidence that these modes are responsible for the transition to the monoclinic phase, nor that the molecule loses its rotational freedom about the fivefold axis in the monoclinic phase.

We have studied the Landau free energy in the rhombohedral phase and calculated the latent heat at the transition to the monoclinic phase. Our numerical value of the latent heat is in good agreement with experiments ${ }^{1,3}$ and molecular dynamics. ${ }^{14}$ The phase transition is found to be of first order. We have thoroughly studied the lattice deformations that are associated with the transition to the monoclinic phase. While the phase transition is mainly an orientational phenomenon, the lattice deformations are coupled with primary and secondary order parameters. The theory accounts qualitatively for the lattice distortions which have been determined by $\mathrm{x}$-ray diffraction experiments. ${ }^{3}$

\section{ACKNOWLEDGMENTS}

This work has been financially supported by the Science Foundation and by the Federal Services for Scientific, Technical and Cultural Affairs of Belgium (Project No. IUAP 48, characterization of materials).

\section{APPENDIX A: SYMMETRY ADAPTED FUNCTIONS}

Here we first quote some relevant site symmetry adapted functions $S_{l}^{\tau}(\Omega)$ of the group $D_{3 d}$. We use the notation of Ref. 26 and define the functions

$$
\begin{gathered}
Y_{l}^{m, c}(\Omega)=\left[Y_{l}^{m}(\Omega)+Y_{l}^{-m}(\Omega)\right] / \sqrt{2}, \\
Y_{l}^{m, s}(\Omega)=-i\left[Y_{l}^{m}(\Omega)-Y_{l}^{-m}(\Omega)\right] / \sqrt{2},
\end{gathered}
$$

For the $A_{1 g}$ representation of $D_{3 d}$ we have the functions $S_{l}^{A_{1 g}, \rho}$, where

$$
\begin{array}{lll}
S_{2}^{A_{1 g}, 1}=Y_{2}^{0}, & S_{4}^{A_{1 g}, 1}=Y_{4}^{0}, & S_{4}^{A_{1 g}, 2}=Y_{4}^{3, s}, \\
S_{6}^{A_{1 g}, 1}=Y_{6}^{0}, & S_{6}^{A_{1 g}, 2}=Y_{6}^{3, s}, & S_{6}^{A_{1 g}, 3}=Y_{6}^{6, c}, \\
S_{8}^{A_{1 g}, 1}=Y_{8}^{0}, & S_{8}^{A_{1 g}, 2}=Y_{8}^{3, s}, & S_{8}^{A_{1 g}, 3}=Y_{8}^{6, c} .
\end{array}
$$

Taking into account relations (A1), (A2), and (2.4) and observing that $Y_{l}^{m *}=Y_{l}^{-m}$ (convention of Ref. 26), we find for the rotator functions $(2.5)$ :

$$
U_{l}^{A_{1 g}, \rho}(\omega)=\sqrt{\frac{4 \pi}{2 l+1}} S_{l}^{A_{1 g}, \rho}(\omega),
$$

where $\omega=(\beta, \alpha)$ are the polar angles of the fivefold molecular axis.

The relevant symmetry-adapted functions which do not belong to the unit representation of $D_{3 d}$ belong to the twodimensional representation $E_{g}$ or $E_{u}$, depending on whether $l$ is even or odd, respectively. We quote

$$
\begin{aligned}
& S_{2}^{E_{g}, 1}=Y_{2}^{1, s}, \quad S_{2}^{E_{g}, 2}=-Y_{2}^{1, c}, \quad S_{2}^{E_{g}, 3}=Y_{2}^{2, c}, \quad S_{2}^{E_{g}, 4}=-Y_{2}^{2, s}, \\
& S_{4}^{E_{g}, 1}=Y_{4}^{1, s}, \quad S_{4}^{E_{g}, 2}=-Y_{4}^{1, c}, \quad S_{4}^{E_{g}, 3}=Y_{4}^{2, c}, \quad S_{4}^{E_{g}, 4}=-Y_{4}^{2, s}, \\
& S_{5}^{E_{u}, 1}=Y_{5}^{1, c}, \quad S_{5}^{E_{u}, 2}=Y_{5}^{1, s}, \quad S_{5}^{E_{u}, 3}=Y_{5}^{2, s}, \quad S_{5}^{E_{u}, 4}=Y_{5}^{2, c}, \\
& S_{5}^{E_{u}, 5}=Y_{5}^{4, s}, \quad S_{5}^{E_{u}, 6}=-Y_{5}^{4, c}, \quad S_{5}^{E_{u}, 7}=Y_{5}^{5, c}, \quad S_{5}^{E_{u}, 8}=-Y_{5}^{5, s} .
\end{aligned}
$$

In the following we will need the rotator function

$$
U_{2}^{E_{g}, 4}(\omega)=-\sqrt{\frac{4 \pi}{5}} S_{2}^{E_{g}, 4}(\omega)
$$

\section{APPENDIX B: ELASTIC CONSTANTS}

Since there are no experimental data on elastic constants of solid $\mathrm{C}_{70}$ in the rhombohedral phase, we have performed model calculations to obtain a reasonable estimation of their values.

As long-range forces due to the attractive van der Waals term of exp-6 potential, Eq. (3.1), are believed to be responsible for bare elastic properties, our calculations involved all neighboring molecules laying inside a sphere centered at a crystal site with radius of $50 \AA$, that is more than five nearest-neighbors' distances. Thus, 161 shells of sites and 639 molecules were included in the process of calculations. The estimated influence from outside molecules was less than $0.09 \%$. Only pair interactions between carbon centers were counted here with the potential parameters of Table I, while double and intermediate interaction centers were omitted due to short-range nature of their interaction. We recall that $D$ and $I$ centers play a significant role in the orientational interactions among nearest neighbors and are counted in the terms such as $V^{R}, V^{\mathrm{RT}}$, and $V^{\mathrm{RRT}}$.

For the present calculations all molecules were fixed in their standard positions and no averages over different orientations were taken into account. Thus, both rotation about long axis and tilting from the $z$ direction were ignored.

The elastic contribution to the crystal energy was calculated at 2401 points as a function of distortions imposed by strain tensor components $\epsilon_{x x}, \epsilon_{y y}, \epsilon_{z z}$, and $\epsilon_{y z}$. As a result we obtained values $a_{\text {calc }}=5.817 \AA, c_{\text {calc }}=9.314 \AA$ of the equilibrium lattice constants close to experimental ones ${ }^{3}$ $a=5.846 \AA, c=9.283 \AA$. The larger theoretical value of $c$ and the smaller value of $a$ are due to the fact that the tilting of molecules was not considered. Our equilibrium volume, $819 \AA^{3}$, is only slightly smaller than the experimentally found value of $824 \AA^{3}$ for the rhombohedral phase. For the calculated elastic constants we obtained the values 


$$
\begin{aligned}
& c_{11}=550.8, \quad c_{12}=72.0, \quad c_{13}=108.5, \quad \mathrm{~K} / \AA^{3}, \\
& c_{14}=58.0, \quad c_{33}=665.0, \quad c_{44}=167.5, \quad \mathrm{~K} / \AA^{3} .
\end{aligned}
$$

If we take the values of the elastic constants of $\mathrm{C}_{60}$ given in Ref. 33 and used in Ref. 12 as a guideline, we conclude that our calculated values Eq. (B1) are too small, by a factor $5-10$.

This situation seems quite typical for molecular-dynamics calculations with bond-charge models which fail to reproduce correct values for the elastic constants. ${ }^{25,33}$ Most likely there exists a long-range multipole contribution arising from a slight redistribution of electric charge inside the $\mathrm{C}_{70}$ molecule, which is not included in pair exp-6 potential. On the other hand, the results on $\mathrm{C}_{60}$ of Ref. 33 have been obtained within a unified model which takes into account tightbinding potential for the intramolecular interaction. Assuming that the omitted long-range interactions are somehow proportional to our potential for the $C-C$ interactions and taking into account the estimated values of the elastic constants for the disordered phase of $\mathrm{C}_{70}$, we took values of the bare elastic constants that are 6 times larger than those given in Eq. (B1):

$$
\begin{gathered}
c_{11}^{0}=3305, \quad c_{12}^{0}=432, \quad c_{13}^{0}=651, \quad \mathrm{~K} / \AA^{3}, \\
c_{14}^{0}=348, \quad c_{33}^{0}=3990, \quad c_{44}^{0}=1005, \quad \mathrm{~K} / \AA^{3}, \\
c_{66}^{0}=\frac{1}{2}\left(c_{11}^{0}-c_{12}^{0}\right)=1436.5, \quad \mathrm{~K} / \AA^{3} .
\end{gathered}
$$

Finally we give relations between elastic constants and Lamé coefficients ${ }^{34}$

$$
\begin{gathered}
c_{11}^{0}=4 \lambda_{\xi \eta ; \xi \eta}+2 \lambda_{\xi \xi ; \eta \eta}, \quad c_{14}^{0}=-2 \lambda_{\xi \xi ; \xi z}, \quad c_{66}^{0}=2 \lambda_{\eta \eta ; \xi \xi}, \\
c_{11}^{0}=4 \lambda_{\xi \eta ; \xi \eta}-2 \lambda_{\xi \xi ; \eta \eta}, \quad c_{33}^{0}=\lambda_{z z ; z z}, \\
c_{13}^{0}=2 \lambda_{\xi \eta ; z z}, \quad c_{44}^{0}=2 \lambda_{\xi z ; \eta z} .
\end{gathered}
$$

*Permanent Address: Institute of Physical Chemistry of RAS, 117915, Moscow, Leninskii prospect 31, Russia.

${ }^{1}$ G.B.M. Vaughan, P.A. Heiney, J.E. Fischer, D.E. Luzzi, D.A. Ricketts-Foot, A.R. McGhie, Y.W. Hui, A.L. Smith, D.E. Cox, W.J. Romanov, B.H. Allen, N. Coustel, J.P. McCauley, Jr., and A.B. Smith III, Science 254, 1350 (1992).

${ }^{2}$ M.A. Verheijen, H. Meekes, G. Meijer, P. Bennema, J.L. de Boer, S. van Smaalen, G. van Tendeloo, S. Amelinckx, S. Muto, J. van Landuyt, Chem. Phys. 166, 287 (1992).

${ }^{3}$ G.B.M. Vaughan, P.A. Heiney, D.E. Cox, J.E. Fischer, D.E. Luzzi, A.R. McGhie, A.L. Smith, R.M. Strongin, M.A. Cichy, and A.B. Smith III, Chem. Phys. 178, 599 (1993).

${ }^{4}$ C. Christides, I.M. Thomas, T.J.S. Dennis, and K. Prassides, Europhys. Lett. 22, 611 (1993).

${ }^{5}$ C. Christides, T.J.S. Dennis, K. Prassides, R.L. Cappelletti, D.A. Neumann, and J.R.D. Copley, Phys. Rev. B 49, 2897 (1994).

${ }^{6}$ C. Meingast, F. Gugenberger, G. Roth, M. Haluška, and H. Kuzmany, Z. Phys. B 95, 67 (1994)

${ }^{7}$ A.R. McGhie, J.E. Fischer, P.A. Heiney, P.W. Stephens, R.L. Cappelletti, D.A. Neumann, W.H. Mueller, H. Mohn, and H.-U. ter Meer, Phys. Rev. B 49, 12614 (1994).

${ }^{8}$ R. Sachidanandam and A.B. Harris, Phys. Rev. Lett. 67, 1467 (1991); A.B. Harris and R. Sachidanandam, Phys. Rev. B 46, 4944 (1992); P.A. Heiney, J.E. Fischer, A.R. McGhie, W.J. Romanov, A.M. Denenstein, J.P. McCauley, Jr., A.B. Smith III, and D.E. Cox, Phys. Rev. Lett. 67, 1468 (1991).

${ }^{9}$ W.I.F. David, R.M. Ibberson, J.C. Matthewman, K. Prassides, T.J.S. Dennis, J.P. Hare, H.W. Kroto, R. Taylor, and D.R.M. Walton, Nature (London) 353, 147 (1991)

${ }^{10}$ K.H. Michel, J.R.D. Copley, and D.A. Neumann, Phys. Rev. Lett. 68, 2929 (1992).

${ }^{11}$ R. Sachidanandam and A.B. Harris, Phys. Rev. B 49, 2878 (1994).

${ }^{12}$ A.K. Callebaut and K.H. Michel, Phys. Rev. B 52, 15279 (1995).

${ }^{13}$ R.M. Lynden-Bell and K.H. Michel, Rev. Mod. Phys. 66, 721 (1994).
${ }^{14}$ M. Sprik, A. Cheng, and M.L. Klein, Phys. Rev. Lett. 69, 1660 (1992).

${ }^{15}$ J. Baker, P.W. Fowler, P. Lazzeretti, M. Malagoli, and R. Zanasi, Chem. Phys. Lett. 184, 182 (1991).

${ }^{16}$ R. Sachidanandam, T.C. Lubenski, and A.B. Harris, Phys. Rev. B 51, 12380 (1995).

${ }^{17}$ W. Que, D.F. Agterberg, and M.B. Walker, Phys. Rev. B 47, 13074 (1993).

${ }^{18}$ D.F. Agterberg and M.B. Walker, Phys. Rev. B 48, 5630 (1993).

${ }^{19}$ D.F. Agterberg, W. Que, and M.B. Walker, Chem. Phys. Lett. 213, 207 (1993).

${ }^{20}$ PH.M. van Loosdrecht, M.A. Verheijen, H. Meekes, P.J.M. van Bentum, and G. Meijer, Phys. Rev. B 47, 7610 (1993).

${ }^{21}$ B.J. Nelissen, P.H.M. van Loosdrecht, M.A. Verheijen, A. van der Avoird, and G. Meijer, Chem. Phys. Lett. 207, 343 (1993).

${ }^{22}$ H.M. James and T.A. Keenan, J. Chem. Phys. 31, 12 (1959).

${ }^{23}$ M. Yvinec and R.M. Pick, J. Phys. (Paris) 41, 1045 (1980).

${ }^{24}$ K.H. Michel and K. Parlinski, Phys. Rev. B 31, 1823 (1985).

${ }^{25}$ A. Cheng and M.L. Klein, Phys. Rev. B 45, 1889 (1992); M. Sprik, A. Cheng, and M.L. Klein, J. Phys. Chem. 96, 2027 (1992).

${ }^{26}$ C.J. Bradley and A.P. Cracknell, The Mathematical Theory of Symmetry in Solids (Clarendon, Oxford, 1972).

${ }^{27}$ K.H. Michel and J.M. Rowe, Phys. Rev. B 32, 5818 (1985); 32, 5827 (1985).

${ }^{28}$ L.D. Landau, Phys. Z. Sowjetunion 11, 26545 (1937).

${ }^{29}$ J.-C. Toledano and P. Toledano, The Landau theory of Phase Transitions (World Scientific, Singapore, 1987).

${ }^{30}$ K.H. Michel and E. Courtens, Phys. Rev. B 23, 513 (1981).

${ }^{31}$ K.H. Michel and K. Parlinski, Phys. Rev. B 31, 1823 (1985).

${ }^{32}$ D. Lamoen and K.H. Michel, Phys. Rev. B 48, 807 (1993).

${ }^{33}$ J. Yu, L. Bi, R.K. Kalia, and P. Vashishta, Phys. Rev. B 49, 5008 (1994).

${ }^{34}$ L.D. Landau and E.M. Lifshitz, Course of Theoretical Physics, Theory of elasticity (Pergamon, Bristol, 1975), Vol. 7. 